\title{
Extremal isolated horizons: a local uniqueness theorem
}

\author{
Jerzy Lewandowski ${ }^{1,2,3}$ and Tomasz Pawlowski ${ }^{1,2}$ \\ ${ }^{1}$ Instytut Fizyki Teoretycznej, Uniwersytet Warszawski, ul. Hoża 69, 00-681 Warsaw, Poland \\ 2 Max Planck Institut für Gravitationsphysik, Albert Einstein Institut, 14476 Golm, Germany \\ 3 Physics Department, 104 Davey, Penn State, University Park, PA 16802, USA
}

Received 15 October 2002

Published 22 January 2003

Online at stacks.iop.org/CQG/20/587

\begin{abstract}
We derive all the axi-symmetric, vacuum and electrovac extremal isolated horizons. It turns out that for every horizon in this class, the induced metric tensor, the rotation 1-form potential and the pullback of the electromagnetic field necessarily coincide with those induced by the monopolar, extremal KerrNewman solution on the event horizon. We also discuss the general case of a symmetric, extremal isolated horizon. In particular, we analyse the case of a two-dimensional symmetry group generated by two null vector fields. Its relevance to the classification of all the symmetric isolated horizons, including the non-extremal ones, is explained.
\end{abstract}

PACS numbers: 04.20.Ex, 04.70.Bw

\section{Introduction}

Isolated horizons. In a new model of black holes in equilibrium [1] the stationarity assumption is relaxed from spacetime and imposed only on an appropriately distinguished null surface. An isolated horizon $(\mathrm{IH})(\triangle,[\ell])$ is a null surface $\Delta$ equipped with a null flow $[\ell]$ which satisfies the following geometric and topological conditions $(\ell$ stands for a null vector field tangent to $\triangle$ and generating the flow). The geometric conditions are:

(i) the (degenerate) metric tensor $q$ induced on $\triangle$ is preserved by the flow $[\ell]$, and

(ii) the same holds for the covariant derivative $\mathbf{D}$, induced ${ }^{4}$ on $\triangle$

$$
£_{\ell} q=0, \quad\left[\mathfrak{f}_{\ell}, \mathbf{D}\right]=0 .
$$

\footnotetext{
${ }^{4}$ The first condition is equivalent to a condition that, for every pair $X, Y$ of vector fields tangent to $\triangle$, the spacetime covariant derivative $\nabla_{X} Y$ is also tangent to $\triangle$. The restriction of $\nabla$ to the tangent bundle of $\Delta$ is the induced covariant derivative $\mathbf{D}$. 
The topological condition is that $\Delta$ contains a space-like 2-surface $\tilde{\triangle}$ homeomorphic to the 2-sphere, and that each orbit is homeomorphic to the line $\mathbb{R}$.

In general, there is no Killing vector field in a neighbourhood of an isolated horizon [6, 2], radiation may exist even arbitrarily close to it. A local description of mechanics and geometry of IHs was developed in [1-3, 7]. One of the structures used by this description is the $I H$ geometry defined by the pair $(q, \mathbf{D})$. If one considers $\triangle$ as a part of the characteristic Cauchy surface for the evolution of the gravitational field possibly coupled to some matter, then, in standard cases (Maxwell, scalar, Yang-Mills field, etc) the pair $(q, \mathbf{D})$ provides the part of the initial data corresponding to the gravitational field. (The pair $(q, \mathbf{D})$ might be thought of as the spacetime metric tensor given at $\Delta$ up to the first order.) IH geometry has local degrees of freedom. The tensor $q$ is arbitrary modulo the requirement that $\ell$ be orthogonal to $\Delta$ and $\mathfrak{f}_{\ell} q=0$. The ingredients of $\mathbf{D}$ are listed in the next section. Here we introduce one of them, namely a 1 -form $\omega$ defined on $\triangle$, such that

$$
\mathbf{D} \ell=\omega \otimes \ell,
$$

and called the rotation 1 -form potential. The existence of $\omega$ follows from $£_{\ell} q=0$. The rotation of the IH is given by $\mathrm{d} \omega$. The surface gravity $\kappa^{(\ell)}$ of the vector field $\ell$ is

$$
\kappa^{(\ell)}=\ell^{a} \omega_{a} .
$$

The surface gravity is constant on $\triangle$,

$$
\kappa^{(\ell)}=\mathrm{const}
$$

owing to the Zeroth Law [1], which holds provided the Ricci tensor $R_{\mu \nu}$ satisfies at $\triangle$

$$
\ell^{a} R_{a \mu} e_{(\triangle)}^{\mu}=0
$$

where the subscript $(\triangle)$ denotes the pullback of a covariant tensor onto $\triangle$. In the EinsteinMaxwell case considered here, this condition follows from the constraint equations we discuss in the next section (in a more general context, this condition is implied by natural energy assumptions [7]). An $\mathrm{IH}(\triangle,[\ell])$ is called extremal, whenever

$$
\kappa^{(\ell)}=0 .
$$

This condition is independent of the choice of generator of the flow $[\ell]$.

The Einstein-Maxwell equations induce constraint equations on the horizon geometry $(q, \mathbf{D})$ and on the electromagnetic field possibly present on $\triangle$. The character of the equations crucially depends on the value of the surface gravity. Whereas the constraints reduce to a system of linear algebraic equations in the non-extremal IH case [7], in the extremal case the constraints become a system of partial differential equations involving the 2-metric $q$, the rotation 1-form $\omega$ and an electromagnetic field $F$ (sections 2.2, 2.3). The general solution is not known. This is the issue we address in this paper.

Our results. The most surprising result contained in this paper follows from our derivation of a general solution of the vacuum and, respectively, electrovac constraints in the axi-symmetric, extremal IH case (section 4). We prove that if the electro-magnetic field $F$ is also axi-symmetric on the horizon and preserved by the null flow $[\ell]$, then $q, \omega$ and the pullback of $F$ onto $\triangle$ coincide with those induced by the monopolar ${ }^{5}$, extremal Kerr-Newman solution on the event horizon. That uniqueness of the Kerr-Newman extremal horizon is surprising, because no non-local condition was imposed except that $\triangle$ contains a slice homeomorphic to the 2-sphere. The question of what distinguishes the Kerr IH in a quasi-local theory was raised in [5] and

\footnotetext{
5 The electromagnetic field depends on one more parameter, the possibly non-zero magnetic charge.
} 
a local characterization of the non-extremal Kerr IH was found. The current result completes that answer.

Other results concern the general, possibly non-symmetric, electrovac, extremal IH case. We study integrability conditions for the constraints and their consequences (sections 2.2, 2.3). In particular, we demonstrate the non-vanishing of certain combinations of the Riemann tensor on $\triangle$. The results are used to prove that, given a null 3-surface in spacetime, there is at most one extremal $\mathrm{IH}$ structure $^{6}$ defined on it, in the vacuum and electrovac cases (section 2.4). That issue was addressed in [7] and our current result completes the discussion of that paper.

The above result is applied to the discussion of the symmetric extremal IHs (section 3). A map $\Delta \rightarrow \triangle$ is called a symmetry, whenever it preserves the geometry $(q, \mathbf{D})$, whereas the flow $[\ell]$ is not necessarily preserved. This definition is motivated by the properties of spacetime isometries: every Killing vector field, if it exists, tangent to a given IH generates its symmetry. An IH that admits a symmetry which is not an element of the flow $[\ell]$ is called symmetric. The cases we analyse are when the symmetry group contains

(i) the $O(2)$ group - the axi-symmetric case already mentioned in this section and, respectively,

(ii) a two-dimensional group of null symmetries - the null-symmetric case.

The null symmetric extremal IHs are also relevant for the classification of all symmetric IHs, including the non-extremal ones. Indeed, all the symmetric non-extremal IHs can be explicitly constructed by using the invariants introduced in [7] except the case when the geometry $(q, \mathbf{D})$ of a non-extremal $\triangle$ admits an extra null symmetry. But then by [7], one of the null generators of the symmetry group defines on the same $\Delta$ another IH structure which is extremal, in the vacuum IH case. We generalize that result to the electrovac case in the current paper.

Conventions. The spacetime signature is $(-,+,+,+)$. By the subscript $(\triangle)$ we denote the pullback of a covariant tensor onto $\triangle$. $\left(e^{\mu}\right)$ and $\left(e_{\nu}\right)$ stand for a coframe of 1 -forms and a frame of vectors, respectively, null, unless indicated otherwise. $e^{1}$ and $e^{2}$ are complex valued, $e^{3}$ and $e^{4}$ are real valued. The spacetime metric is

$$
g=e^{1} \otimes e^{2}+e^{2} \otimes e^{1}-e^{3} \otimes e^{4}-e^{4} \otimes e^{3} .
$$

Due to the symmetry of every IH $\triangle$, all the considerations will automatically descend to the sphere $\hat{\Delta}$ of the null geodesics in $\triangle$. We will not distinguish between the functions defined on $\hat{\triangle}$ and the functions defined on $\triangle$ which are constant along the null geodesics.

\section{The constraint equations}

In this section, we discuss the constraint equations satisfied by the geometry $(q, \mathbf{D})$ of an $\mathrm{IH}(\triangle,[\ell])$ in the vacuum and electrovac cases, respectively and their integrability conditions. The constraints are given by that part of the spacetime energy-momentum tensor $T_{\mu \nu}$ that can be derived from $(q, \mathbf{D})$ via the Einstein equations, that is by the pullback of $T_{\mu \nu}$ onto $\triangle$. The stress-energy tensor is constructed from an electro-magnetic field present on $\triangle$. We add to the system of equations, the constraints on the electromagnetic field $F$ on $\triangle$ following from the Maxwell equations. The symmetry assumption is that the flow $[\ell]$ is a symmetry of the electromagnetic field on $\triangle$. An arbitrary surface gravity is used in the equations to indicate

6 Of course, a generic null surface admits no IH structure. 
the essential difference between the extremal and non-extremal cases. The non-extremal case will also be considered in section 3.3.

An IH $(\triangle,[\ell])$ is called a vacuum $I H$ whenever the spacetime Ricci tensor $R_{\mu v}$ satisfies on $\triangle$

$$
\left(R_{\mu \nu} e^{\mu} \otimes e^{\nu}\right)_{(\triangle)}=0 .
$$

In the presence of an electromagnetic field $F$ at an $\mathrm{IH}(\triangle,[\ell])$, the part of the Einstein equations we assume on $\triangle$ is the pullback

$$
\left(\left(R_{\mu \nu}-8 \pi T_{\mu \nu}\right) e^{\mu} \otimes e^{\nu}\right)_{(\triangle)}=0,
$$

where $T_{\mu \nu}$ is the gravitational energy-momentum tensor of the electromagnetic field, which satisfies in particular $T_{\mu}^{\mu}=0$. The Maxwell equations define extra constraints on the components of the electromagnetic field $F$ on $\triangle$. We make a small effort now, to write them in a geometric form. One of the constraints on $F$ at $\triangle$ is

$$
\ell\lrcorner \star(d(F-\mathrm{i} \star F))=0,
$$

where we used the spacetime Hodge star. For the differential forms defined on $\triangle$ and transversal to $\ell$, there is a well-defined intrinsic Hodge dual ${ }^{(\Delta)} \star$ (see the appendix). The remaining constraint equation on $F$ is

$$
\left({ }^{(\triangle)} \star-\mathrm{i}\right)\left[(\star d(F-\mathrm{i} \star F))_{(\triangle)}\right]=0 .
$$

We will also assume that the electromagnetic field is invariant with respect to the null flow $[\ell]$. This assumption reads

$$
£_{\ell}(F-\mathrm{i} \star F)_{\nu}^{\mu} e_{(\triangle)}^{\nu} \otimes e_{\mu}=0
$$

where the above Lie derivative is well defined for the following reasons. It follows from the equations (9) and the vanishing of the expansion of $\ell$ that

$$
T_{a b} \ell^{a} \ell^{b}=0
$$

A consequence of this fact is

$$
\ell\lrcorner(F-\mathrm{i} \star F)_{(\triangle)}=0 .
$$

Therefore, if we consider the tensor $(F-\mathrm{i} \star F)_{v}{ }^{\mu}$ as a 1 -form taking vector values, then its pullback on $\triangle$ takes values in the space $T(\triangle)$ tangent to $\triangle$.

Concluding, an electrovac isolated horizon is an $\mathrm{IH}(\triangle,[\ell])$ which admits an electromagnetic field $F$ such that the constraint equations (9)-(11), (14) and the symmetry condition (12) are satisfied. The electromagnetic field $F$ will be referred to as an electromagnetic field that makes $\triangle$ an electrovac $I H$.

Equation (14) is the condition which implies that $\ell^{a} T_{a \mu} e_{(\Delta)}^{\mu}=0$, the condition ensuring the Zeroth Law (4) via the constraint equations.

\subsection{Geometry of an IH and the spacetime Ricci tensor}

Ingredients of $(q, \mathbf{D})$ and $\left.F_{(} \Delta\right)$ in an adapted frame. Before we turn to the constraints on $(q, \mathbf{D})$ in detail, we specify the elements of $(q, \mathbf{D})$ which are free before imposing the constraints. It follows from the definition that an isolated horizon $\Delta$ can be viewed as a cylinder $\hat{\triangle} \times \mathbb{R}$ generated by null geodesics where the projection

$$
\Pi: \triangle=\hat{\Delta} \times \mathbb{R} \rightarrow \hat{\Delta}
$$


maps each null geodesic into a point of the topological sphere $\hat{\triangle}$. The induced metric tensor is

$$
q=g_{(\triangle)}
$$

where $g$ is a spacetime metric.

The assumption

$$
\mathfrak{f}_{\ell} q=0
$$

implies that $q$ is a pullback by $\Pi$ of a 2 -metric tensor $\hat{q}$ defined on $\hat{\triangle}$,

$$
q=\Pi^{*} \hat{q} .
$$

Let us fix a null vector field $\ell$ tangent to $\triangle$ and representing the flow [ $\ell]$. It is also convenient to fix a foliation of $\triangle$ by 2 -cross-sections (called 'slices' hereafter) of the projection $\Pi$ preserved by the flow $[\ell]$. Then, a null vector field $n$ transversal to $\Delta$ is uniquely defined by assuming the orthogonality to the slices and the normalizing condition

$$
n_{\mu} \ell^{\mu}=-1 \text {. }
$$

The covariant derivative $\mathbf{D}$ consists of the following components:

(i) the metric connection defined on any slice by the 2-metric $q$,

(ii) the rotation 1 -form potential $\omega$,

(iii) the deformations of the induced metric tensor on a 2-slice while it is transported along the orthogonal null vector field $n$.

Let $\left(e_{1}, e_{2}, e_{3}, e_{4}\right)=(m, \bar{m}, n, \ell)$ be a null frame defined in a spacetime neighbourhood of $\triangle$. The degenerate metric tensor $q$ induced on $\triangle$ is

$$
q=\left(e^{1} \otimes e^{2}+e^{2} \otimes e^{1}\right)_{(\triangle)} .
$$

The real vectors $\operatorname{Re}(m), \operatorname{Im}(m)$ are (automatically) tangent to $\triangle$. To adapt the frame further, we assume the vector fields $\operatorname{Re}(m), \operatorname{Im}(m)$ are tangent to the slices of the fixed foliation. We can choose the vector field $m$ such that

$$
\mathfrak{£}_{\ell} m=0,
$$

hence, the projection of $m$ onto $\hat{\triangle}$ uniquely defines a null frame $\hat{m}$ tangent to $\hat{\triangle}$,

$$
\Pi_{*} m=: \hat{m} \text {. }
$$

The frame $\left(e^{1}, e^{2}, e^{3}, e^{4}\right)$ is adapted to: the vector field $\ell$, the $[\ell]$ invariant foliation of $\triangle$, and the null complex-valued frame $\hat{m}$ defined on the sphere $\triangle$. Spacetime frames constructed in this way on $\triangle$ will be called adapted.

The elements of an adapted frame are Lie dragged by $\ell$,

$$
£_{\ell} e_{(\triangle)}^{\mu}=0 .
$$

Therefore, the connection components defined by $\mathbf{D}$ in that frame are constant along the null geodesics tangent to $\ell$. They are

$$
\begin{aligned}
& m^{v} \mathbf{D} \bar{m}_{v}=\Pi^{*}\left(\hat{m}^{A} \hat{\nabla} \overline{\hat{m}}_{A}\right)=: \Pi^{*} \hat{\Gamma}, \\
& -n_{\nu} \mathbf{D} \ell^{v}=\omega=\pi e_{(\triangle)}^{2}+\bar{\pi} e_{(\triangle)}^{1}+\kappa^{(\ell)} e_{(\triangle)}^{3}, \\
& -\bar{m}^{v} \mathbf{D} n_{v}=\mu e_{(\triangle)}^{1}+\lambda e_{(\triangle)}^{2}+\pi e_{(\triangle)}^{4}, \\
& m_{\mu} \mathbf{D} \ell^{\mu}=0,
\end{aligned}
$$


where $\hat{\Gamma}$ is the Levi-Civita connection 1-form corresponding to the covariant derivative $\hat{\nabla}$ defined by $\hat{q}$ and to the null frame $\hat{m}$ defined on $\hat{\Delta}$.

Due to $\left[\mathfrak{f}_{\ell}, \mathbf{D}\right]=0$,

$$
\mathfrak{f}_{\ell} \omega=0 .
$$

Therefore, there is a 1 -form $\hat{\omega}$ defined on $\triangle$, such that

$$
\Pi^{*} \hat{\omega}=\pi e_{(\Delta)}^{2}+\bar{\pi} e_{(\Delta)}^{1} .
$$

In the case of an extremal $\mathrm{IH}, \hat{\omega}$ is uniquely defined on $\Delta$ by $[\ell]$ and $\mathbf{D}$.

The global existence of solutions to the constraint equations on $\Delta$ will be crucial for us, hence we conclude this subsection with a discussion of the global existence of the objects defined above. Defined globally on $\Delta$ are: the vector field $\ell=e_{4}$, the covector $e_{(\Delta)}^{4}$, the function $\mu$, the tensor $\lambda e^{2} \otimes e^{2}$, the 1-forms $\omega$ and $\hat{\omega}$, and the constant $\kappa^{(\ell)}$. It will be convenient to express the 1-form $\hat{\omega}$ by two functions $U$ and $\ln B$ globally defined on $\hat{\Delta}$ (sufficiently many times differentiable) using the well-known decomposition

$$
\hat{\omega}=\hat{\star} \mathrm{d} U+\mathrm{d} \ln B
$$

where $\hat{\star}$ is Hodge star defined by the 2 -metric tensor $\hat{q}$. The coefficient $\pi$ of $\hat{\omega}$ becomes

$$
\pi=-\mathrm{i} \bar{\delta} U+\bar{\delta} \ln B .
$$

The Ricci tensor components present in the constraints. We turn now to the pullback of the spacetime Ricci tensor onto $\Delta$ and express it by $(q, \mathbf{D})$. We still admit a possibly non-zero surface gravity $\kappa^{(\ell)}$ both because the non-extremal case will be considered in the last section, and to emphasize the difference between the extremal and non-extremal cases.

Due to $\mathfrak{£}_{\ell} q=0, \mathfrak{f}_{\ell} \omega=0, d \kappa^{(\ell)}=0$ and $\left[\mathfrak{f}_{\ell}, \mathbf{D}\right]=0$ the Ricci tensor already satisfies

$$
\ell^{a} R_{a \mu} e_{(\Delta)}^{\mu}=0, \quad £_{\ell}\left(R_{\mu \nu} e^{\mu} \otimes e^{\nu}\right)_{(\Delta)}=0 .
$$

The remaining components of the pullback onto $\Delta$ of the Ricci tensor will be expressed in terms of the following objects defined on the sphere $\hat{\Delta}$ : the null frame $(\hat{m}, \overline{\hat{m}})$ and the dual coframe $\left(\hat{e}^{1}, \hat{e}^{2}\right)$, the complex-valued differential operator

$$
\delta:=\hat{m}^{A} \partial_{A},
$$

(where $A=1,2$ refers to coordinate system $\left(x^{1}, x^{2}\right)$ defined locally on $\hat{\Delta}$ ), the connection form $\hat{\Gamma}$ defined above

$$
\hat{\Gamma}=: 2 \bar{a} \hat{e}^{1}+2 a \hat{e}^{2},
$$

its curvature scalar

$$
K:=2 \delta a+2 \bar{\delta} \bar{a}-8 a \bar{a},
$$

and the 1 -form $\hat{\omega}$ and its divergence

$$
\operatorname{div} \hat{\omega}=\delta \pi+\bar{\delta} \bar{\pi}-2 a \bar{\pi}-2 \bar{a} \pi .
$$

The spacetime Ricci tensor components in question are

$$
\begin{aligned}
& R_{m \bar{m}}=2 \kappa^{(\ell)} \mu-\operatorname{div} \hat{\omega}-\hat{\omega}^{2}+K, \\
& R_{\bar{m} \bar{m}}=2 \kappa^{(\ell)} \lambda-2 \bar{\delta} \pi-4 a \pi-2 \pi^{2},
\end{aligned}
$$

where $\hat{\omega}^{2}:=\hat{\omega}_{A} \hat{\omega}_{B} \hat{q}^{A B}$. As we mentioned before, the components have to be constant along the null geodesics. Note that the remaining connection components $\mu$ and $\lambda$ are not involved in the formula for the pullback of the Ricci tensor. 


\subsection{The constraints in the extremal vacuum case}

We consider now an extremal, vacuum IH; that is, we assume equations (6), (8) hold on $\triangle$. The constraints amount to the following equations on the metric $\hat{q}$ and the 1 -form $\hat{\omega}$ defined on the sphere $\hat{\triangle}$ :

$$
\begin{aligned}
& \operatorname{div} \hat{\omega}+\hat{\omega}^{2}-K=0, \\
& \bar{\delta} \pi+2 a \pi+\pi^{2}=0 .
\end{aligned}
$$

This system of equations can also be written in the following compact, tensorial form

$$
\hat{\nabla}_{(A} \hat{\omega}_{B)}+\hat{\omega}_{A} \hat{\omega}_{B}-\frac{1}{2} \hat{R}_{A B}=0,
$$

where $\hat{\nabla}$ and $\hat{R}_{A B}$ are the covariant derivative and Ricci tensor defined on the sphere $\hat{\triangle}$ by the 2-metric $\hat{q}$.

In terms of the functions $U$ and $B$ defined in (27), (28), equations (35a), (35b) read

$$
\begin{aligned}
& (\delta \bar{\delta}+\bar{\delta} \delta-2 a \delta-2 \bar{a} \bar{\delta}+2 \mathrm{i} \delta U \bar{\delta}-2 \mathrm{i} \bar{\delta} U \delta+2 \delta U \bar{\delta} U-K) B=0, \\
& (\bar{\delta} \bar{\delta}+2 a \bar{\delta}-2 \mathrm{i} \bar{\delta} U \bar{\delta}-\mathrm{i} \bar{\delta} \bar{\delta} U-2 \mathrm{i} a \bar{\delta} U-\bar{\delta} U \bar{\delta} U) B=0
\end{aligned}
$$

where by $\delta U$ or $\delta \delta U$, etc we mean functions, not the product of the operators. Note that the equations do not involve the real-valued scalar $\mu$ and the complex-valued tensor $\lambda \hat{e}^{2} \otimes \hat{e}^{2}$, which are therefore freely defined on $\hat{\triangle}$. An equivalent system of equations was considered in [7]. An integrability condition given by commuting the $\delta$ operator with $\delta \bar{\delta}+\bar{\delta} \delta$ as well as $\bar{\delta}$ with $\delta \delta$ is

$$
\bar{\delta} \Psi_{2}+3 \pi \Psi_{2}=0,
$$

where $\Psi_{2}$ is an invariant which characterizes the geometry $(q$, D) of a vacuum IH,

$$
\Psi_{2}=\frac{1}{2}(-K+\mathrm{i}(\delta \bar{\delta}+\bar{\delta} \delta-2 a \delta-2 \bar{a} \bar{\delta}) U) .
$$

From the spacetime point of view, the function $\Psi_{2}$ is the spinorial component $C_{\ell n \ell n}-C_{\ell n m \bar{m}}$ of the Weyl tensor $C_{\mu \nu \alpha \beta}$, it is always constant along the null generators of $\triangle$ and the integrability condition (38) is one of the Bianchi identities. The condition implies the following identity via (28),

$$
\Psi_{2} B^{3} \mathrm{e}^{-3 \mathrm{i} U}=C_{0}=\text { const. }
$$

Since $B$ nowhere vanishes (by definition (27)), and $K \neq 0$ on a non-trivial subset of $\hat{\triangle}$, we have

$$
C_{0} \neq 0
$$

The solution for $B$ is

$$
B=B_{0}\left(\Psi_{2}\right)^{-\frac{1}{3}} \mathrm{e}^{\mathrm{i} U}
$$

where $B_{0} \neq 0$ is a constant. The conditions

$$
B=\bar{B}>0
$$

imply constraints on the remaining unknowns, that is on the metric tensor $q$ and the function $U$. Another consequence of (41) that will be important in section 2.4 is the following nonvanishing lemma:

Lemma 1. For every extremal, vacuum IH the invariant $\Psi_{2}$ nowhere vanishes on $\triangle$ :

$$
\Psi_{2}(x) \neq 0
$$

for every $x \in \triangle$. 


\subsection{The constraints in the extremal electrovac case}

Now we consider an extremal, electrovac IH; that is, we assume that equations (6), (9)(12) hold on $\triangle$. Let $F=\frac{1}{2} F_{\mu \nu} e^{\mu} \wedge \epsilon^{\nu}$ be an electromagnetic field present in a spacetime neighbourhood of the horizon. We use the standard notation for the components of $F$ in a null frame,

$$
F=-\Phi_{0} e^{4} \wedge e^{1}+\Phi_{1}\left(e^{4} \wedge e^{3}+e^{2} \wedge e^{1}\right)-\Phi_{2} e^{3} \wedge e^{2}+\text { c.c. }
$$

Condition (14) implied by the constraints reads

$$
\Phi_{0}=0 .
$$

The assumed invariance of $F_{\nu}{ }^{\mu} e_{(\Delta)}^{v} \otimes e_{\mu}$ with respect to the null flow [ $\left.\ell\right]$ is equivalent to the requirement that the coefficients $\Phi_{1}$ and $\Phi_{2}$ be constant along the null geodesics generating $\triangle$.

Now, the part of the constraints (10), (11) coming from the Maxwell equations amounts to the following equation on $\Phi_{1}$ only,

$$
\bar{\delta} \Phi_{1}+2 \pi \Phi_{1}=0
$$

which is easy to integrate into the following form:

$$
\Phi_{1}=E_{0} B^{-2} \mathrm{e}^{2 \mathrm{i} U},
$$

$E_{0}$ being a complex constant, and the functions $U$ and $B$ being defined by (28).

The part of the constraints following from the Einstein equations (9) becomes

$$
R_{m m}=0, \quad R_{m \bar{m}}=4\left|\Phi_{1}\right|^{2} .
$$

In terms of the geometry $\hat{q}$ of the sphere $\hat{\triangle}$ and the 1 -form $\hat{\omega}$, the constraints read

$$
\hat{\nabla}_{(A} \hat{\omega}_{B)}+\hat{\omega}_{A} \hat{\omega}_{B}-\frac{1}{2} \hat{R}_{A B}+2\left|\Phi_{1}\right|^{2} \hat{q}_{A B}=0 .
$$

Finally, after expressing $\hat{\omega}$ by the functions $U$ and $B$, the constraints read

$$
\begin{aligned}
& (\delta \bar{\delta}+\bar{\delta} \delta-2 a \delta-2 \bar{a} \bar{\delta}+2 \mathrm{i} \delta U \bar{\delta}-2 \mathrm{i} \bar{\delta} U \delta+2 \delta U \bar{\delta} U-K) B+4 \frac{\left|E_{0}\right|^{2}}{B^{3}}=0 \\
& (\bar{\delta} \bar{\delta}+2 a \bar{\delta}-2 \mathrm{i} \bar{\delta} U \bar{\delta}-\mathrm{i} \bar{\delta} \bar{\delta} U-2 \mathrm{i} a \bar{\delta} U-\bar{\delta} U \bar{\delta} U) B=0 .
\end{aligned}
$$

Concluding, an extremal IH $(\triangle,[\ell])$ is an extremal, electrovac IH if and only if it admits an electromagnetic field $F$ (45) such that the components $\Phi_{1}, \Phi_{2}$ are constant along the null geodesics in $\triangle$ and the geometry $(q, \mathbf{D})$ and $F$ satisfy equations (46), (48) and (51). Note that the components $\mu, \lambda$ of $\mathbf{D}$ as well as the component $\Phi_{2}$ of $F$ are not involved in the equations, and $\Phi_{1}$ is a complex-valued function defined globally on the sphere $\hat{\triangle}$. The assumption that $\Phi_{2}$ is constant along the null geodesics in $\triangle$ was used to eliminate the derivatives $\ell^{a} \partial_{a} \Phi_{2}$ from the Maxwell equations and derive the equation (47).

We complete this subsection by a discussion of the consequences of the constraint equations. It follows from (48) that:

Lemma 2. The component $\Phi_{1}$ of the electromagnetic field either vanishes nowhere on $\triangle$, or it is zero everywhere on $\triangle$. In the second case, $\triangle$ is a vacuum isolated horizon (even if $\Phi_{2} \neq 0$ ).

Commuting the $\delta$ operator with $\delta \bar{\delta}+\bar{\delta} \delta$ as well as $\bar{\delta}$ with $\delta \delta$ we derive the following integrability conditions for the equations (51)

$$
(\bar{\delta}+3 \pi) \Psi_{2}-\pi R_{m \bar{m}}=0,
$$


where as before, $\Psi_{2}$ is the component $C_{4343}-C_{4312}$ of the Weyl tensor, and in the presence of the electromagnetic field it takes on $\triangle$ the following value:

$$
\Psi_{2}=2 \frac{\left|E_{0}\right|^{2}}{B^{4}}+\frac{1}{2}(-K+\mathrm{i}(\delta \bar{\delta}+\bar{\delta} \delta-2 a \delta-2 \bar{a} \bar{\delta}) U)
$$

The integrability condition is one of the Bianchi identities.

In this case, we were not able to solve completely the integrability condition for the functions $\Psi_{2}$ and $B$. However, the fact of non-vanishing of $\Psi_{2}$ on an extremal vacuum IH can be generalized in the following way (applied later in section 2.4):

Lemma 3. For every extremal electrovac IH $\triangle$, there is an open and dense subset $\mathcal{V} \subset \triangle$ such that

$$
3 \Psi_{2}-\left.R_{m \bar{m}}\right|_{\mathcal{V}} \neq 0
$$

Proof. In the vacuum case the conclusion of the lemma was already proved, hence we prove it now assuming that $R_{m \bar{m}}$ is not identically zero on $\triangle$. As a consequence, $R_{m \bar{m}}$ is nowhere zero; that is, for every point $x \in \triangle$,

$$
R_{m \bar{m}}(x) \neq 0 \text {. }
$$

Using equations (48) and (52) we derive the following equation

$$
\bar{\delta}\left(\left(3 \Psi_{2}-R_{m \bar{m}}\right) B^{3} \mathrm{e}^{-3 i U}\right)+16\left|E_{0}\right|^{2} \mathrm{e}^{-3 \mathrm{i} U} \bar{\delta} B^{-1}=0,
$$

where

$$
E_{0} \neq 0
$$

due to the assumption (55). Let $\mathcal{U}$ be an open subset of $\triangle$ on which

$$
3 \Psi_{2}-R_{m \bar{m}}=0 .
$$

It follows from (56) that

$$
\left.B\right|_{\mathcal{U}}=\text { const } \neq 0 \text {. }
$$

Then, equation $(51 a)$ takes the following form:

$$
-K+R_{m \bar{m}}+\left.2|\bar{\delta} U|^{2}\right|_{\mathcal{U}}=0 .
$$

Moreover, it follows from (58) that

$$
\left.K\right|_{\mathcal{U}}=\frac{1}{3} R_{m \bar{m}},
$$

hence, we have finally

$$
R_{m \bar{m}}+\left.3|\bar{\delta} U|^{2}\right|_{\mathcal{U}}=0
$$

Since each of the terms is non-negative, they both vanish on $\mathcal{U}$. This contradiction completes the proof.

\subsection{Uniqueness of the electrovac extremal $\mathrm{IH}$}

Given an extremal IH $(\triangle,[\ell])$ and its geometry $(q, \mathbf{D})$ a natural question is whether there exists another null flow

$$
\left[\ell^{\prime}\right] \neq[\ell]
$$

such that $\left(\triangle,\left[\ell^{\prime}\right]\right)$ is an extremal $\mathrm{IH}$ as well. Suppose then that there exists a function $f$ defined on $\triangle$ such that

$$
\ell^{\prime}=f \ell, \quad\left[\mathfrak{\ell}_{\ell^{\prime}}, \mathbf{D}\right]=0, \quad \mathbf{D}_{\ell^{\prime}} \ell^{\prime}=0 .
$$


The first two equations are equivalent to the following equation [7]:

$$
\left(\mathbf{D}_{a} \mathbf{D}_{b}+2 \omega_{(a} \mathbf{D}_{b)}\right) f=0 .
$$

The last equation in (64) implies that the function $f$ is constant along the null geodesics tangent to $\ell$,

$$
\ell^{a} \partial_{a} f=0
$$

The integrability condition for (65) obtained by acting on it with $\mathbf{D}_{a}$ can be expressed by the spacetime curvature components [7]

$$
\left(3 \Psi_{2}-R_{m \bar{m}}\right) \bar{\delta} f=0,
$$

where we have already assumed that $\Delta$ is an electrovac IH. Owing to lemma 3 , the condition above implies $f=$ const, and

$$
\left[\ell^{\prime}\right]=\ell \text {. }
$$

Proposition 1. If $(\triangle,[\ell]$ ) is an extremal electrovac IH (including the vacuum case) and a null vector field $\ell^{\prime}$ tangent to $\triangle$ satisfies

$$
\mathfrak{£}_{\ell^{\prime}} q=0, \quad\left[\mathfrak{£}_{\ell^{\prime}}, \mathbf{D}\right]=0, \quad D_{\ell^{\prime}} \ell^{\prime}=0,
$$

then $\left[\ell^{\prime}\right]=[\ell]$.

In conclusion, given a null surface $\Delta$ in a spacetime, there is at most one extremal $\mathrm{IH}[\ell]$ defined thereon.

\section{Symmetric IHs}

Given an IH $(\triangle, \ell)$ suppose that there is a vector field $X$ tangent to $\triangle$, such that

$$
\mathfrak{£}_{X} q=0=\left[\mathfrak{£}_{X}, \mathbf{D}\right] .
$$

We call $X$ a generator of a symmetry of the geometry $(q, \mathbf{D})$. Obviously, the vector field $\ell$ is a symmetry itself. If $X \notin[\ell]$, then we call $\Delta$ a symmetric IH. Note that it is not assumed that the symmetries preserve $[\ell]$. Nonetheless they do so, however, at least in the extremal, electrovac or vacuum case, due to proposition 1.

Corollary 1. Given an electrovac extremal $I H(\triangle,[\ell])$, suppose X is a generator of a symmetry of the geometry $(q, \mathbf{D})$. Then, the flow $[X]$ preserves the flow $[\ell]$, that is

$$
[X, \ell]=a_{0} \ell, \quad a_{0}=\text { const. }
$$

In this section we discuss two cases of symmetric extremal isolated horizons: the axisymmetric case and the null-symmetric case. Since the geometry $(q, \mathbf{D})$ of a null-symmetric extremal electrovac IH admits also a non-extremal IH structure, for completeness we formulate in the last subsection a converse statement.

\subsection{Axisymmetric extremal IHs}

Consider an extremal IH $(\triangle,[\ell])$ whose geometry $(q, \mathbf{D})$ has a symmetry group isomorphic to $\mathrm{O}(2)$ and which satisfies the conclusion of corollary 1 . We show now that in this case, the symmetry group $O(2)$ commutes with the flow $[\ell]$, and we adapt coordinates on $\triangle$. The constraint equations will be solved in the next section. 
Label the elements of the group by a parameter, $[0,2 \pi] \ni \varphi \mapsto U(\varphi)$ such that

$$
U\left(\varphi_{1}\right) U\left(\varphi_{2}\right)=U\left(\varphi_{1}+\varphi_{2}\right), \quad U(0)=U(2 \pi)=\text { identity. }
$$

According to corollary 1 , a vector field $U(\varphi)_{*} \ell$ defines the same IH as $\ell$, hence

$$
U(\varphi)_{*} \ell=a_{0}(\varphi) \ell,
$$

where $a_{0}(\varphi) \in \mathbb{R}$ is a constant. As a function of $\varphi$, it satisfies

$$
a_{0}\left(\varphi_{1}\right) a_{0}\left(\varphi_{2}\right)=a_{0}\left(\varphi_{1}+\varphi_{2}\right), \quad a_{0}(0)=a_{0}(2 \pi)=1 .
$$

The only solution is

$$
U(\varphi)_{*} \ell=\ell .
$$

Therefore, the following is true.

Proposition 2. Suppose $(\triangle,[\ell])$ is an extremal, electrovac IH; suppose further a vector field $\Phi$ tangent to $\triangle$ is a generator of a proper symmetry group of the IH geometry, and the group it generates is isomorphic to $O(2)$. Then

$$
£_{\ell} \Phi=0 \text {. }
$$

Given an axisymmetric IH, we adjust coordinates on $\triangle$ in the following way. Note first that due to proposition 2 the projection $\Pi_{*} \Phi$ defines uniquely a vector field on $\hat{\triangle}$,

$$
\hat{\boldsymbol{\Phi}}:=\Pi_{*} \Phi
$$

and the first equality in (70) implies that

$$
£_{\hat{\Phi}} \hat{q}=0 .
$$

Therefore $\hat{\boldsymbol{\Phi}}$ indeed generates a group of rotations of $\hat{q}$. It follows that there are spherical coordinates $(\theta, \varphi)$ on $\hat{\triangle}$ naturally pulled back to $\triangle$, and an extra coordinate $v$ on $\triangle$, such that

$$
\boldsymbol{\Phi}=a(\theta, \varphi) \partial_{v}+\partial_{\varphi},
$$

modulo a constant factor. To kill the first term locally, it is enough to replace $v$ by a new coordinate

$$
v^{\prime}=v-A(\theta, \varphi), \quad A(\theta, \varphi):=\int_{\varphi_{0}(\theta)}^{\varphi} a\left(\theta, \varphi^{\prime}\right) .
$$

The resulting $v^{\prime}$ is a continuous function defined globally on $\triangle$, due to the condition

$$
\int_{\varphi_{0}}^{\varphi_{0}+2 \pi} a(\theta, \varphi)=0
$$

which follows from the assumption that the orbits of $\Phi$ are closed and from the fact that each orbit can intersect each null geodesics in $\triangle$ only once (otherwise, the orbit would have a self-intersection). The differentiability of $v^{\prime}$ may be ensured by appropriate choice of the boundary of integration $\varphi_{0}(\theta)$ as a function of $\theta$. In the coordinates $\left(v^{\prime}, \theta, \varphi\right)$, the vector field $\Phi$ is

$$
\boldsymbol{\Phi}=\partial_{\varphi}
$$

Instead of the auxiliary coordinate $\theta$ on $\hat{\triangle}$, used implicitly above, we introduce a 'Hamiltonian' function, naturally defined by the vector field $\hat{\Phi}$ and the area 2 -form $\hat{\epsilon}$ of $\hat{q}$, namely a function $x$ such that

$$
\hat{\Phi}\lrcorner \hat{\epsilon}=2 \mathrm{~d} x
$$


The global existence on $\hat{\triangle}$ and the differentiability of this function is ensured by the fact that

$$
d(\hat{\Phi}\lrcorner \hat{\epsilon})=£_{\hat{\Phi} \hat{\epsilon}}=0 .
$$

The function $x$ has exactly two extremal points, a minimum and a maximum, at the two zero points of $\hat{\Phi}$, and the additive constant is fixed by the condition

$$
\sup x=\frac{A}{8 \pi}, \quad \inf x=-\frac{A}{8 \pi},
$$

where $A$ is a constant, equal to the area of $\hat{\triangle}$. Concluding, we are going to use the functions $(\varphi, x)$ as spherical coordinates on $\hat{\triangle}$, and, denoting in the same way their pullbacks to $\triangle$, the functions $\left(v^{\prime}, \varphi, x\right)$ as coordinates on $\triangle$. Adjusting the 2-frame $\hat{m}$ on $\hat{\triangle}$ such that

$$
£_{\hat{\Phi}} \hat{m}=0,
$$

we get the adapted frame $\left(e^{1}, e^{2}, e^{3}, e^{4}\right)$ whose pullbacks onto $\triangle$ are invariant with respect to the rotations generated by $\Phi$.

\subsection{Null-symmetric extremal IHs}

An IH is called null-symmetric if it admits a generator $X$ of a symmetry, such that

$$
q_{a b} X^{a} X^{b}=0, \quad X \notin[\ell] .
$$

Suppose this is the case. There exists a function $f$ defined on $\triangle$, such that

$$
X=f \ell,
$$

and the equations (65) hold. It follows from them that

$$
\mathbf{D}_{b} \ell^{a} \partial_{a} f=0 \text {. }
$$

If $v$ is a function defined on $\triangle$, such that

$$
\ell^{a} \partial_{a} v=1,
$$

then the general solution of (89) is

$$
f=b+\kappa_{0} v, \quad \ell^{a} \partial_{a} b=0, \quad \kappa_{0}=\text { const } \neq 0 .
$$

The case of $\kappa_{0}=0$ is excluded by proposition 1 . Note that every linear combination $X-a_{0} \ell, a_{0}$ a constant, defines a non-extremal IH $(\triangle,[X])$ (that is, the null surface $\triangle$ admits many distinct IHs). Therefore [7], every cross section of $\Delta \rightarrow \hat{\triangle}$ given by the equation

$$
b+\kappa_{0} v=\text { const, }
$$

has identically vanishing shear and expansion in each null direction orthogonal to this cross section. In other words, if we use a null frame $(m, \bar{m}, n, \ell)$ adapted to the foliation of $\triangle$ given by (92), then the following coefficients of $\mathbf{D}$ vanish (see (24c))

$$
\mu=\lambda=0 \text {. }
$$

The converse is also true. Suppose an extremal IH $(\triangle,[\ell])$ admits a foliation by 2 -spacelike surfaces such that every null vector field tangent to any leaf has zero shear and expansion on $\triangle$. Then [7] $\triangle$ is a null-symmetric IH. The proper null symmetry is generated by the vector field

$$
X=v \ell,
$$

where $v$ is a function constant on the leaves and such that $\ell^{a} v_{,_{a}}=1$. 
Even though there are still some degrees of freedom left in the geometry of a nullsymmetric extremal IH $(\triangle,[\ell])$, the group of the null symmetries is always the same in the electrovac case. We characterize this group now.

It follows from (91) that the Lie algebra defined by the vector fields $\ell$ and $X$ above is unique, namely given by the following commutator:

$$
\left[\ell, \frac{1}{\kappa_{0}} X\right]=\ell \text {. }
$$

Suppose $X^{\prime}$ is another null generator of a null symmetry. We will see it belongs to the Lie algebra spanned by $\ell$ and $X$. Indeed, as before

$$
X^{\prime}=\left(b^{\prime}+\kappa_{0}^{\prime} v\right) \ell, \quad \ell^{a} \partial_{a} b^{\prime}=0, \quad \kappa_{0}^{\prime} \neq 0 .
$$

Consider the following linear combination of the generators:

$$
Y:=\frac{1}{\kappa_{0}} X-\frac{1}{\kappa_{0}^{\prime}} X^{\prime}
$$

It is also a null generator of a symmetry of $(q, \mathbf{D})$. Note that

$$
Y=\left(\frac{1}{\kappa_{0}} b-\frac{1}{\kappa_{0}^{\prime}} b^{\prime}\right) \ell=b^{\prime \prime} \ell, \quad \ell^{a} \partial_{a} b^{\prime \prime}=0 .
$$

Therefore,

$$
\mathbf{D}_{Y} Y=0 \text {. }
$$

Owing to proposition 1 , if $(\triangle,[\ell])$ is an extremal electrovac $\mathrm{IH}$, then there is a constant $a_{0}^{\prime}$ such that

$$
Y=a_{0}^{\prime} \ell
$$

In conclusion,

Proposition 3. A general null-symmetric electrovac (including vacuum) extremal $I H(\triangle,[\ell]$ ) is given by any solution $(\hat{q}, \hat{\omega})$ of the constraint equations $(51 a),(51 b)$ and $\mu=0=\lambda$ in (24c). Its group of the null symmetries is exactly two-dimensional, the generators are a vector field $\ell \in[\ell]$ and $X=v \ell$ where $v$ is a function constant on the foliation corresponding to $\mu$ and $\lambda$, and such that $\ell^{a} v_{, a}=1$. The commutator between the generators is

$$
[\ell, X]=\ell \text {. }
$$

\subsection{Null-symmetric non-extremal IHs}

As noted above, a null-symmetric, extremal, electrovac IH admits a null vector field which defines on the same null surface a non-extremal IH. In this subsection, we discuss a converse statement.

Consider a non-extremal IH $(\triangle,[\ell])$ such that there exists on $\triangle$ a null vector field $X$ whose flow is different from $[\ell]$ and which generates a symmetry of the IH geometry $(q, \mathbf{D})$. If $(\triangle,[\ell])$ is a vacuum $\mathrm{IH}$, then [7] there is another vector field $\ell^{\prime}$ tangent to $\triangle$, such that $\left(\triangle,\left[\ell^{\prime}\right]\right)$ is an extremal null-symmetric IH characterized above. We generalize now this result to the electrovac case. (Our proof will also be valid for the vacuum case. In this way we fill a small gap in the proof sketched in [7].) The vector field $X$ can be written as

$$
X=f \ell,
$$

where the function $f$ satisfies the equations (65). Those equations imply that

$$
f=b \mathrm{e}^{-\kappa^{(\ell)} v}+a_{0}
$$


where $a_{0}$ is a constant, $\kappa^{(\ell)} \neq 0$ is the surface gravity of $\ell$,

$$
\ell^{a} \partial_{a} v=1, \quad \ell^{a} \partial_{a} b=0,
$$

and the function $b$ satisfies the following equations

$$
\begin{aligned}
& {\left[\frac{1}{2}(\delta \bar{\delta}+\bar{\delta} \delta-(\alpha-\bar{\beta}) \delta-(\bar{\alpha}-\beta) \bar{\delta})+\pi \delta+\bar{\pi} \bar{\delta}+\mu \kappa^{(\ell)}\right] b=0,} \\
& {\left[(\delta+\bar{\alpha}-\beta+2 \bar{\pi}) \delta+\bar{\lambda} \kappa^{(\ell)}\right] b=0 .}
\end{aligned}
$$

The resulting vector field satisfies

$$
\mathbf{D}_{X} X=a_{0} \kappa^{(\ell)} X \text {. }
$$

Therefore, if $b$ nowhere vanishes, then $X$ corresponding to $a_{0}=0$ defines an extremal IH on $\triangle$. We show the non-vanishing of the function $b$ assuming that $(\triangle,[\ell])$ admits an electromagnetic field $F$ of the properties which make $\triangle$ an electrovac IH, and such that

$$
£_{f \ell}\left(F_{\mu}{ }^{\nu} e^{\mu} \otimes e_{\nu}\right)=0 .
$$

The integrability condition for the equations (105) has the following form

$$
\left(3 \Psi_{2}-R_{m \bar{m}}\right) \bar{\delta} b+\left((\bar{\delta}+3 \pi) \Psi_{2}\right) b=0,
$$

whereas (107) implies

$$
\Phi_{2} \ell^{a} \partial_{a} f-2 \Phi_{1} \bar{\delta} f=0 .
$$

The part of Maxwell equations used in the definition of the electrovac isolated horizon allows us to express $\Phi_{2}$ by $\Phi_{1}$ :

$$
\kappa^{(\ell)} \Phi_{2}=\bar{\delta} \Phi_{1}+2 \pi \Phi_{1} .
$$

Then, equation (109) for $f$ given by (103) becomes

$$
\left(\bar{\delta} \Phi_{1}+2 \pi \Phi_{1}\right) b+2 \Phi_{1} \bar{\delta} b=0 .
$$

This constraint can easily be integrated and is equivalent to

$$
\Phi_{1} b^{2} \mathrm{e}^{-2 \mathrm{i} U}=E_{0}=\text { const. }
$$

If $E_{0}$ is not zero then $b$ cannot vanish, therefore we assume that

$$
E_{0}=0 \text {. }
$$

Let

$$
\mathcal{U}=\{x \in \triangle: b(x) \neq 0\} .
$$

Since

$$
\left.\Phi_{1}\right|_{\mathcal{U}}=0
$$

the condition (108) implies that the following complex-valued function $C$ given by

$$
C:=\Psi_{2} b^{3} \mathrm{e}^{-3 \mathrm{i} U},
$$

is constant on $\mathcal{U}$. Suppose $C \neq 0$ on a connected component of $\mathcal{U}$. If it is not the entire sphere $\hat{\triangle}$, then there is a sequence $x_{n} \in \mathcal{U}, n=1,2,3, \ldots$ such that

$$
x_{n} \rightarrow x_{0} \quad \text { and } \quad b\left(x_{0}\right)=0 .
$$

But then

$$
\left|\Psi_{2}\left(x_{n}\right)\right| \rightarrow \infty
$$


Hence, the only possibility for $b$ to vanish somewhere on $\triangle$ is

$$
\left.C\right|_{\mathcal{U}}=0,\left.\quad \Psi_{2}\right|_{\mathcal{U}}=0
$$

In the non-extremal case we can always choose a foliation by space-like cross sections, preserved by $\ell$, such that

$$
\pi=-\mathrm{i} \bar{\delta} U \text {. }
$$

Given that foliation, consider on $\hat{\triangle}$ a 1 -form

$$
\beta:=\partial\left(\mathrm{e}^{\mathrm{i} U} b\right):=\frac{\partial}{\partial z}\left(\mathrm{e}^{-2 \mathrm{i} U} b\right) \mathrm{d} z,
$$

where $z$ is any local holomorphic coordinate on $\hat{\triangle}$. Then the vanishing of $\Psi_{2}$ and equation (105a) imply

$$
\bar{\partial}\left(\mathrm{e}^{-2 \mathrm{i} U} \beta\right)=0
$$

everywhere on $\hat{\triangle}$. On a sphere, the only solution to this equation is

$$
\beta=0=\partial\left(\mathrm{e}^{\mathrm{i} U} b\right) \text {. }
$$

And that implies

$$
b=\text { const }
$$

on the entire $\triangle$. Since we assumed $\left[\left(b \mathrm{e}^{-\kappa^{(\ell)} v}+a_{0}\right) \ell\right] \neq[\ell]$, the conclusion is that

$$
b(x) \neq 0 \quad \text { for every } \quad x \in \triangle,
$$

and the vector field

$$
\ell^{\prime}=b \mathrm{e}^{-\mathrm{i} v} \ell
$$

defines an extremal IH on $\triangle$. Our discussion is summarized by the following:

Proposition 4. Suppose a non-extremal IH $(\triangle,[\ell])$ admits a null vector field $X \notin[\ell]$ which generates a symmetry of the IH geometry. If $\triangle$ admits an electromagnetic field $F$ which makes it an electrovac $\mathrm{IH}$, and such that

$$
£_{X}\left(F_{\mu}{ }^{v} e^{\mu} \otimes e_{\nu}\right)_{(\triangle)}=0,
$$

then $(\triangle,[X])$ is an extremal IH.

\section{General axisymmetric solution: the uniqueness of the monopolar extremal Kerr-Newman IH}

In this section, we consider an axi-symmetric extremal IH $(\triangle,[\ell])$ and an electromagnetic field $F$ such that

$$
£_{\Phi}(F-\mathrm{i} \star F)_{\nu}{ }^{\mu} e_{(\triangle)}^{\nu} \otimes e_{\mu}=0,
$$

where $\Phi$ is a generator of the axial symmetry of $\triangle$. We solve completely the constraint equations (9)-(12) imposed on the geometry $(q, \mathbf{D})$ and on the pullback $F_{(\Delta)}$. First, we find all the local solutions. Next we select those that can be defined globally on $\Delta=\hat{\triangle} \times \mathbb{R}$. Finally, we identify the solutions by using their area, and electric and magnetic charges as corresponding to those defined by the extremal Kerr-Newman solution (of the electromagnetic field modified by the allowed magnetic charge). 


\subsection{General solution of the constraints}

The local constraints. In the coordinates $(\varphi, x)$, the null 2 -frame on $\hat{\triangle}$ takes the following form:

$$
\hat{m}=\frac{1}{2}\left(\frac{1}{P} \partial_{x}+\mathrm{i} P \partial_{\varphi}\right), \quad \hat{e}^{1}=\left(P \mathrm{~d} x-\mathrm{i} \frac{1}{P} \mathrm{~d} \varphi\right) .
$$

Due to the rotational symmetry, the function $P$ and all the functions used in the constraint equations are functions of the variable $x$ only. For this frame, the connection coefficient $a$ of (31) is

$$
a=\frac{P_{x}}{4 P^{2}} .
$$

The constraints (51) on the functions $P, U, B, \Phi_{1}$ read

$$
\begin{aligned}
& \left(\frac{B}{P}\right)_{x x}+\left(P_{x}\right)^{2} \frac{B}{P}+8 P \frac{\left|E_{0}\right|^{2}}{B^{3}}=0 \\
& B_{x x}-\left(U_{x}\right)^{2} B=0, \quad\left(U_{x} B^{2}\right)_{x}=0,
\end{aligned}
$$

where the second and the third equations above, are the real and the imaginary parts of the constraint $(51 b)$ (the third one being extra multiplied by $B$ ). The gauge freedom is

$$
U \mapsto U+U_{0}, \quad B \mapsto B_{0} B,
$$

whereas the freedom in the choice of the coordinates is already eliminated by the choice of the coordinate $x$.

The global existence conditions. The global existence conditions are that the metric tensor

$$
\hat{q}:=\hat{e}^{1} \otimes \hat{e}^{2}+\hat{e}^{2} \otimes \hat{e}^{1}
$$

derived from the function $P$ and the scalar functions $U, B, \Phi_{1}$ are globally defined on $\hat{\triangle}$ and differentiable as many times as one needs, and that $B$ are nowhere zero on $\hat{\triangle}$ and the metric tensor $\hat{q}$ is nowhere degenerate.

In the consideration below, the global existence conditions will be applied in the following way. We will first find local solutions of the constraint equations (51); subsequently we will find $B, U, \Phi_{1}$ and $P$ well defined everywhere on $\hat{\triangle}$ except the poles $x=x_{ \pm}:= \pm \frac{A}{8 \pi}$. Next, we will apply the following condition for the coordinate function $P$ at the poles, necessary for the metric $\hat{q}$ to be of the class $C^{1}$,

$$
\begin{aligned}
& \lim _{x \rightarrow x_{ \pm}} \frac{1}{P}=0, \\
& \lim _{x \rightarrow x_{ \pm}}\left(\frac{1}{P^{2}}\right)_{x}=\mp 2 .
\end{aligned}
$$

Integration. We start with the equations $(131 b),(131 c)$. The integration of the second one gives

$$
U_{x} B^{2}=\tilde{\Omega}=\text { const. }
$$

Note that $\tilde{\Omega}=0$ if and only if the horizon $\triangle$ is non-rotating,

$$
\tilde{\Omega}=0 \quad \Leftrightarrow \quad \mathrm{d} \hat{\omega}=0 \quad \Leftrightarrow \quad \mathrm{d} \omega=0 .
$$


Consider the rotating case first,

$$
\omega \neq 0 \text {. }
$$

We use (135) to eliminate the function $U$ from the first equation in $(131 b),(131 c)$, which now reads

$$
B_{x x}-\frac{\tilde{\Omega}^{2}}{B^{3}}=0
$$

The general solution for $B$ is

$$
B=B_{0}\left(\Omega^{2}+\left(x-x_{0}\right)^{2}\right)^{\frac{1}{2}}, \quad \Omega=\frac{\tilde{\Omega}}{B_{0}^{2}},
$$

where $B_{0}$ and $x_{0}$ are arbitrary constants. Using the integral (135) again we find $U$,

$$
U=\arctan \left(\frac{x-x_{0}}{\Omega}\right)+U_{0}, \quad U_{0}=\text { const. }
$$

In this way we have exhausted the vanishing of the Ricci tensor component $R_{m m}$, and found both of the potentials defining the rotation 1-form potential $\omega$. But to know the 1 -form $\omega$ itself we need the frame operator given by $P$. To find $P$ we turn to the equation $(51 a)$ with the above solutions substituted for $U$ and $B$ and $\Phi_{1}$ given by (48),

$\left[\frac{\mathrm{d}^{2}}{\mathrm{~d} x^{2}}+\frac{2\left(x-x_{0}\right)}{\left(x-x_{0}\right)^{2}+\Omega^{2}} \frac{\mathrm{d}}{\mathrm{d} x}+\frac{4 \Omega^{2}}{\left(\left(x-x_{0}\right)^{2}+\Omega^{2}\right)^{2}}\right] \frac{1}{P^{2}}=-\frac{16\left|E_{0}\right|^{2}}{B_{0}{ }^{4}\left(\left(x-x_{0}\right)^{2}+\Omega^{2}\right)^{2}}$.

The general solution is

$$
\frac{1}{P^{2}}=\frac{c_{1}\left(\Omega^{2}-\left(x-x_{0}\right)^{2}\right)+2 c_{2} \Omega\left(x-x_{0}\right)-\frac{8\left|E_{0}\right|^{2}}{B_{0}{ }^{4}}}{\Omega^{2}+\left(x-x_{0}\right)^{2}} .
$$

The requirement that $P$ be finite on the open interval $x \in]-\frac{A}{8 \pi}, \frac{A}{8 \pi}[$, and satisfy the conditions (134) at the poles determines the values of the constants $x_{0}, c_{1}, c_{2}, \frac{\left|E_{0}\right|^{2}}{B_{0}{ }^{4}}$.

The solution. In the rotating case, the resulting solutions $P, U, B, \Phi_{1}$ of the MaxwellEinstein equations (47), (51) on $\triangle$ can be expressed by three real parameters $A, \alpha$ and $\theta_{0}$ of the domain of dependence specified below, where the third one is a constant phase of $\Phi_{1}$ and does not affect the horizon geometry $(q, \mathbf{D})$ :

$$
\begin{aligned}
& P^{2}(x)=\frac{4 \pi\left(1+\alpha^{2}\right)}{A} \frac{A^{2}+(8 \pi)^{2} \frac{1-\alpha^{2}}{1+\alpha^{2}} x^{2}}{A^{2}-(8 \pi)^{2} x^{2}} \\
& U= \pm \arctan \left(8 \pi \sqrt{\frac{1-\alpha^{2}}{1+\alpha^{2}}} \frac{x}{A}\right) \\
& B=\left(1+\frac{1-\alpha^{2}}{1+\alpha^{2}} \frac{(8 \pi x)^{2}}{A^{2}}\right)^{\frac{1}{2}}, \\
& \Phi_{1}=\mathrm{e}^{\mathrm{i} \theta_{0}} \frac{2 \sqrt{\pi} A^{\frac{3}{2}} \alpha}{1+\alpha^{2}} \frac{\left(A^{2}-\frac{1-\alpha^{2}}{1+\alpha^{2}}(8 \pi x)^{2}\right) \pm 2 \mathrm{i} A \sqrt{\frac{1-\alpha^{2}}{1+\alpha^{2}}}(8 \pi x)}{\left(A^{2}+\frac{1-\alpha^{2}}{1+\alpha^{2}}(8 \pi x)^{2}\right)^{2}}, \\
& \alpha \in\left[0,1[, \quad A \in] 0, \infty\left[, \quad \theta_{0} \in[0,2 \pi[.\right.\right.
\end{aligned}
$$

Above we have fixed the gauge freedom in the rescaling of $B$ by a constant and adding a constant to $U$ such that as $\alpha \rightarrow 1$ the function $B$ has a finite limit and $U$ goes to zero. 
In the non-rotating case, we take the constant $\tilde{\Omega}$ in (135) to be zero,

$$
\tilde{\Omega}=0,
$$

and repeat all the steps of the derivation above including solving the conditions (134) at the poles. The result is a family of solutions parametrized by a real constant $A>0$,

$$
\begin{aligned}
& P^{2}(x)=\frac{8 \pi A}{A^{2}-(8 \pi x)^{2}} \\
& U=0 \\
& B=1 \\
& \Phi_{1}=\mathrm{e}^{\mathrm{i} \theta_{0}}\left(\frac{\pi}{A}\right)^{\frac{1}{2}} .
\end{aligned}
$$

It turns out that the non-rotating solutions coincide with $P, U, B, \Phi_{1}$ obtained by substituting the parameter $\alpha$ in the rotating case for the excluded values \pm 1 . (Locally, the non-rotating case then has as solutions those obtained by the limit $\alpha \rightarrow 1$.)

\subsection{The uniqueness of the extremal Kerr-Newman IH}

The solutions ( $\left.q, \omega, F_{(\triangle)}\right)$ are given by (24b), (26), (28), (45), (46), (129), (143), (146) and set up a three-parameter family. To compare the solutions with those given by the Kerr-Newman metrics, we use the horizon area, and the electromagnetic charges. The electric and magnetic charges are given by the real and, respectively, the imaginary part of the following integral:

$$
\frac{1}{4 \pi} \int_{\hat{\Delta}} * F+\mathrm{i} F=\mathrm{e}^{\mathrm{i} \theta_{0}} \alpha \sqrt{\frac{A}{4 \pi}} .
$$

The condition that magnetic charge vanishes implies

$$
\mathrm{e}^{\mathrm{i} \theta_{0}}= \pm 1
$$

Therefore, in the absence of magnetic charge, the derived family of $\left(q, \omega, F_{(\triangle)}\right)$ is globally and uniquely parametrized by the area $A$ of (a slice of) the horizon and the electric charge $Q$ contained therein, having the following domain of dependence,

$$
A \in] 0, \infty], \quad Q \in\left[-\sqrt{\frac{A}{4 \pi}}, \sqrt{\frac{A}{4 \pi}}\right] .
$$

But for each pair $(A, Q)$ of (149) there is the extremal Kerr-Newman metric and the corresponding electromagnetic field defining an isolated horizon of area $A$ and electric charge $Q$. Therefore, our family of $\left(q, \omega, F_{(\triangle)}\right)$ consists of the ones given by the extremal KerrNewman solutions. In conclusion,

Theorem 1. Suppose $(\triangle,[\ell])$ is an extremal isolated horizon and $F_{\mu}^{v} e_{(\triangle)}^{\mu} \otimes e_{\nu}$ is an electromagnetic field defined on $\triangle$, such that the flow $[\ell]$ is a symmetry of the electromagnetic field and the total magnetic charge of $\triangle$ is zero. Suppose also that both the IH geometry and the electromagnetic field admit a symmetry group of motions of $\triangle$ isomorphic to $O(2)$. Then, the Einstein-Maxwell constraints (9)-(11) are satisfied if and only if the induced metric q, the rotation potential 1-form $\omega$ and the pullback of the electromagnetic field 2-form $F_{(\triangle)}$ coincide with those defined by the extremal Kerr-Newman solution on the event horizon. Admitting a non-zero magnetic charge does not affect the possible geometries $(q, \mathbf{D})$. 
Recall that, after solving the constraints, the remaining components of the induced connection $\mathbf{D}$, that is the transversal expansion $\mu$, and transversal shear $\lambda\left(e^{2} \otimes e^{2}\right)_{(\Delta)}$ of the lives of a preserved by the symmetries foliation, are an arbitrary real function and a complex-valued tensor, respectively, satisfying the symmetry conditions

$$
\begin{aligned}
& \ell^{a} \partial_{a} \mu=0=\Phi^{\mathbf{a}} \partial_{\mathbf{a}} \mu, \\
& £_{\ell}\left(\lambda e^{2} \otimes e^{2}\right)_{(\triangle)}=0=£_{\Phi}\left(\lambda e^{2} \otimes e^{2}\right)_{(\triangle)} .
\end{aligned}
$$

Similarly, the component $\Phi_{2}$ of the electromagnetic field has to satisfy the symmetry assumptions, whereas the Einstein-Maxwell equations do not impose any extra constraint on it at $\triangle$. Despite this arbitrariness, the symmetries of those remaining components affected the form of the constraints which were solved above. For completeness, the full extremal Kerr-Newman geometry $(q, \mathbf{D})$ is given by the following $\mu$ and $\lambda$ :

$$
\begin{aligned}
& \mu=-\frac{\sqrt{\frac{8 \pi}{1+\alpha^{2}}} A^{\frac{3}{2}}}{A^{2}+\frac{1-\alpha^{2}}{1+\alpha^{2}}(8 \pi x)^{2}}, \\
& \lambda=-\frac{\sqrt{\frac{8 \pi}{1+\alpha^{2}}} A^{\frac{1}{2}} \frac{1-\alpha^{2}}{1+\alpha^{2}}\left(A^{2}-(8 \pi x)^{2}\right)}{\left(A^{2}+\frac{1-\alpha^{2}}{1+\alpha^{2}}(8 \pi x)^{2}\right)\left(A \mp \mathrm{i} \sqrt{\frac{1-\alpha^{2}}{1+\alpha^{2}}}(8 \pi x)\right)},
\end{aligned}
$$

modulo the choice of the foliation of $\triangle$.

\section{Acknowledgments}

We would like to thank Abhay Ashtekar, Jiri Bicak, Piotr Chrusciel, Jim Isenberg, Jacek Jezierski, Vince Moncrief and Bernd Schmidt for discussions and Josh Willis for careful reading of the paper. This work was supported in part by the Polish Committee for Scientific Research (KBN) under grant no 2 PO3B 12724, the Eberly research funds of Penn State and the Albert Einstein Institute of the Max Planck Society.

\section{Appendix. The intrinsic Hodge dual}

The intrinsic Hodge dual we used in (11) is defined on $\triangle$ as follows. An orientation and time orientation of spacetime are used to define an orientation of space-like 2-surfaces of $\triangle$. A neighbourhood of a 2 -surface $\hat{\triangle}$ is diffeomorphic to $\hat{\triangle} \times \mathbb{R} \times \mathbb{R}$ where the factors $\mathbb{R}$ correspond to the null geodesics orthogonal to $\hat{\triangle}$ : the first factor the ones transverse to $\triangle$ and the second to those contained in $\triangle$. The factors $\mathbb{R}$ are oriented according to the time orientation. Then the orientation of $\hat{\triangle}$ is such that the orientation of $\hat{\triangle} \times \mathbb{R} \times \mathbb{R}$ agrees with the orientation of spacetime. Next, we introduce on $\Delta$ the 2-volume form [1] ${ }^{(2)} \epsilon$, a 2-form such that the area of any 2 -surface $\hat{\triangle} \subset \Delta$ is $\int_{\hat{\Delta}}{ }^{(2)} \epsilon$. Given a 1 -form $\mu$ defined on $\triangle$, such that $\ell^{a} \mu_{a}=0$, we define

$$
{ }^{(2)} * \mu_{a}={ }^{(2)} \epsilon_{a b} q^{b c} \mu_{c}
$$

where $q^{a b}$ is such that $q_{a b} q^{b c} v_{c}=v_{a}$ for every 1-form $v$ orthogonal to $\ell$. 


\section{References}

[1] Ashtekar A, Beetle C and Fairhurst S 2000 Class. Quantum Grav. 17 253-98 (Preprint gr-qc/9907068)

[2] Ashtekar A, Beetle C, Dreyer O, Fairhurst S, Krishnan B, Lewandowski J and Wiśniewski J 2000 Phys. Rev. Lett. 85 3564-7 (Preprint gr-qc/0006006)

[3] Ashtekar A, Beetle C and Lewandowski J 2001 Phys. Rev. D 64044016 (Preprint gr-qc/0103026)

[4] Mars M 2000 Class. Quantum Grav. 173353 (Preprint gr-qc/0004018)

[5] Lewandowski J and Pawlowski T 2002 Int. J. Mod. Phys. D 11 739-46 (Preprint gr-qc/0101008)

[6] Lewandowski J 2000 Class. Quantum Grav. 17 L53-9 (Preprint gr-qc/9907058)

[7] Ashtekar A, Beetle C and Lewandowski J 2002 Class. Quantum Grav. 19 1195-225 (Preprint gr-qc/0111067)

[8] Chandrasekhar S 1983 The Mathematical Theory of Black Holes (Oxford: Oxford University Press) 